\title{
Can cardiovascular risk management be improved by shared care with general practice to prevent cognitive decline following stroke/TIA? A feasibility randomised controlled trial (SERVED memory)
}

William J. Davison', Phyo K. Myint ${ }^{2}$, Yoon K. Loke 3 , Garth Ravenhill ${ }^{4}$, David Turner ${ }^{3}$, Chris Fox ${ }^{3}$, Lee Shepstone ${ }^{3}$ and John F. Potter ${ }^{1 *}$ (D)

\begin{abstract}
Background: Cognitive impairment and dementia following cerebrovascular disease are increasingly common in the UK. One potential strategy to prevent post-stroke cognitive decline is multimodal vascular risk factor management. However, its efficacy remains uncertain and its application in vulnerable patients with incident cerebrovascular disease and early cognitive impairment has not been assessed.

The primary aim of this study was to assess the feasibility of recruitment and retention of patients with early cognitive impairment post-stroke or transient ischaemic attack (TIA) to a trial of enhanced vascular risk factor management combining primary and secondary care.

Methods: In this single centre, open label trial adults with a recent stroke or TIA and mild cognitive impairment (MCI) were randomised 1:1 to a three-monthly multimodal vascular risk factor intervention jointly delivered by the trial team and General Practitioner (GP), or control (defined as usual care from the GP). Chosen risk factors were blood pressure (BP), total cholesterol, blood glucose ( $\mathrm{HbA1C}$ ) in those with diabetes, and heart rate and adequacy of anticoagulation in those with atrial fibrillation (AF). Similar patients with normal cognition were enrolled in an embedded observational cohort and also received usual care from the GP. Repeat cognitive screening was undertaken in all participants after 12 months.

(Continued on next page)
\end{abstract}

\footnotetext{
*Correspondence: john.potter@uea.ac.uk

${ }^{1}$ Ageing and Stroke Medicine, Norwich Medical School, University of East Anglia, Bob Champion Research Building, James Watson Road, Norwich, UK Full list of author information is available at the end of the article
}

C C The Author(s). 2020 Open Access This article is licensed under a Creative Commons Attribution 4.0 International License, which permits use, sharing, adaptation, distribution and reproduction in any medium or format, as long as you give appropriate credit to the original author(s) and the source, provide a link to the Creative Commons licence, and indicate if changes were made. The images or other third party material in this article are included in the article's Creative Commons licence, unless indicated otherwise in a credit line to the material. If material is not included in the article's Creative Commons licence and your intended use is not permitted by statutory regulation or exceeds the permitted use, you will need to obtain permission directly from the copyright holder. To view a copy of this licence, visit http://creativecommons.org/licenses/by/4.0/. The Creative Commons Public Domain Dedication waiver (http://creativecommons.org/publicdomain/zero/1.0/) applies to the data made available in this article, unless otherwise stated in a credit line to the data. 
(Continued from previous page)

Results: Seventy three participants were recruited to the randomised trial and 94 to the observational cohort (21.8\% of those screened). From the randomised trial 35/73 (47.9\%) dropped out before final follow-up. In all groups guideline based rates of risk factor control were mostly poor at baseline and did not significantly improve during follow-up. The observational cohort demonstrated greater decline in cognitive test scores at 12 months, with no difference between the randomised groups.

Conclusions: Recruitment to such a study was feasible, but retention of participants was difficult and generally poor rates of risk factor control suggested insufficient application of the intervention. Consequently, successful scaling up of the trial would require protocol changes with less reliance on primary care services. Any future trial should include participants with normal cognition post-stroke as they may be at greatest risk of cognitive decline.

Trial registration: ISRCTN, ISRCTN42688361. Registered 16 April 2015.

Keywords: Cognitive impairment, Dementia after stroke, Vascular dementia, Stroke, Cerebrovascular disease

\section{Background}

Dementia is a significant and increasing health problem in the UK, yet disease modifying treatments are lacking [1], therefore strategies to prevent cognitive decline are desirable. Given that cognitive impairment may affect up to $40 \%$ of patients following stroke and TIA [2-4], such strategies may be particularly valuable in this patient group. One potential strategy is multimodal vascular risk factor control as these risk factors contribute to recurrent stroke as well as both vascular dementia $(\mathrm{VaD})$ and Alzheimer's disease [5-7], and their presence also increases the risk of early cognitive decline progressing to dementia [8]. Evidence supports the value of good BP control for reducing the risk of subsequent severe cognitive impairment post-stroke, yet there remains uncertainty about the value of targeting other vascular risk factors that are relevant to secondary stroke recurrence, especially as part of a multimodal risk factor approach $[5,9,10]$. Furthermore, whether targeting such a strategy at patients who already have MCI post-stroke in order to prevent further cognitive deterioration has not been studied [11-13].

SERVED Memory (Screening and Enhanced Risk factor management to prevent Vascular Event related Decline in Memory) was developed to investigate the feasibility of recruiting patients with MCI post-stroke or TIA to a pragmatic intervention trial of enhanced vascular risk factor management. It was hypothesised that enhanced risk factor management with a "treat to target" approach, delivered by a combination of the patient's GP and a trial team, would be safe and effective, potentially reducing the risk of progression of $\mathrm{MCI}$ compared to standard GP management alone. The trial also incorporated an embedded non-randomised observational cohort with the aim of providing epidemiological data regarding the natural history of cognitive impairment post-stroke or TIA.

\section{Methods}

SERVED Memory was a single-centre, open-label parallel group randomised controlled feasibility trial, with embedded non-randomised observational cohort. The trial was granted ethical approval and was prospectively registered (ISRCTN 42688361). The full trial protocol has previously been published [14]. This report adheres to the CONSORT guidelines for pilot and feasibility trials.

In brief, participants were recruited from stroke services at the Norfolk and Norwich University Hospital (NNUH). Adults with a mild stroke or TIA within the last 8 weeks and Montreal Cognitive Assessment (MoCA) score $\geq 26$ were eligible for the observational cohort, and those with a MoCA score consistent with MCI (i.e. 20-25 [11, 12]) were eligible for the randomised controlled trial (RCT). Patients with life expectancy $<1$ year, diagnosed depression, or MoCA score $<20$ were excluded. All participants provided written informed consent. RCT participants were randomised 1:1 by computer generated randomisation table, with block size of four, to an intervention or control group. Enrolment, randomisation, and intervention allocation were undertaken by the trial nurses or research fellow. Baseline recording of demographic data, medication use and compliance, and vascular risk factors was completed. Measured risk factors were clinic BP, total cholesterol, blood glucose HbA1c in those with diabetes, and heart rate and anticoagulation adequacy for those with AF. Targets were ideal BP $<130 / 80 \mathrm{mmHg}$ and standard < $140 / 90 \mathrm{mmHg}[15,16]$; total cholesterol $<4.0 \mathrm{mmol} / \mathrm{L}$ (non-fasting); HbA1C 48-53 mmol/mol; heart rate $60-80$ beats per minute for those in AF. Adequate anticoagulation was defined as taking warfarin with INR 2.5-3.0, or a direct oral anticoagulant, unless contraindicated. Observation and control participants received usual care from their GP only. Intervention participants were seen in hospital by the trial team at 3, 6, and 9 months postrandomisation for risk factor assessment. Results were 
passed immediately to the GP for action by phone and letter with the trial team only making treatment alterations when necessary for patient safety. All participants were followed up at 12 months for assessment of risk factors, medication adherence, adverse events and repeat MoCA. Baseline frailty was retrospectively assessed from clinical notes using the Rockwood Frailty Score by a stroke physician blinded to group allocation.

The primary outcome was the assessment of rates of recruitment and retention at 12 months from screening and management logs. Secondary outcomes were (i) rates of risk factor control to the specified targets in each group (ii) differences in the change in MoCA score between the intervention and control groups, (iii) change in MoCA score in the observational arm, and (iv) rates of adverse events (including recurrent stroke) in each group.

A convenience sample size was based on estimates of the prevalence of cognitive impairment in patients with incident stroke/TIA [4], the incidence of dementia poststroke [17], and estimated cognitive screening rates at $\mathrm{NNUH}$ [4]. Based on these estimates target numbers were 100 in the RCT (50 per group) and 100 in the observational cohort.

\section{Statistical analysis}

Data were analysed using SPSS (version 25.0) with descriptive statistics only unless specified. Screening logs were assessed to determine the proportion of eligible participants who consented to participate in the trial, including the proportion that would have been eligible for the RCT. Management logs were assessed for retention rates in each trial arm and, where possible, reasons for attrition were identified. Proportions of participants with controlled risk factors in each group were calculated at baseline and follow-up along with the frequency of medication changes that occurred during the trial. Changes in MoCA score from baseline to follow-up for each arm were assessed using a paired samples $t$ test, with further testing of any difference between the intervention and control arms. A general linear model, with a normal error term, was used to estimate the effect of the intervention, with a $95 \%$ confidence interval, on the 12 month MoCA values. The model included randomisation group (intervention or control), sex, diagnosis (stroke or TIA) and baseline MoCA value. Post-hoc analysis of the difference in baseline frailty score in retained vs. not retained participants was assessed with a Mann-Whitney U test.

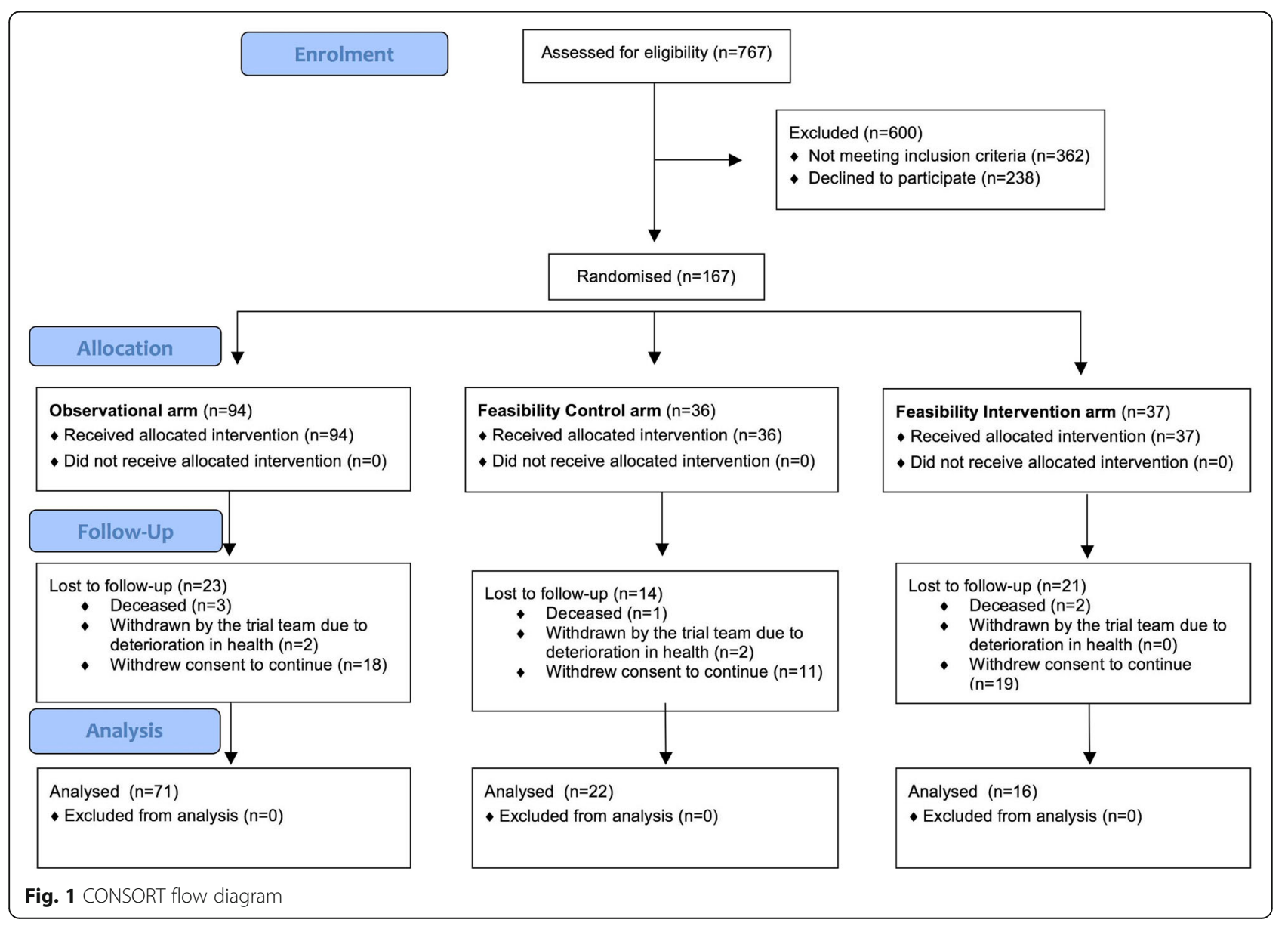


Table 1 Demographic data for each group at baseline

\begin{tabular}{|c|c|c|c|c|}
\hline & & Observation & Control & Intervention \\
\hline $\bar{N}$ & & 94 & 36 & 37 \\
\hline Symptom onset to randomisation (days) & & $25.7(20.1)$ & $22.6(20.9)$ & $17.8(19.7)$ \\
\hline Age (years) & & $72.1(10.9)$ & $74.9(9.2)$ & $75.0(12.0)$ \\
\hline Sex (male) & & $59(62.8 \%)$ & $23(63.9 \%)$ & $27(73.0 \%)$ \\
\hline Ethnicity (White British) & & $94(100.0 \%)$ & $36(100.0 \%)$ & $37(100.0 \%)$ \\
\hline \multirow[t]{3}{*}{ Smoking status } & Non-smoker & $38(40.4 \%)$ & $17(47.2 \%)$ & $26(70.3 \%)$ \\
\hline & Ex-smoker & $29(30.9 \%)$ & $14(38.9 \%)$ & $10(27.0 \%)$ \\
\hline & Current smoker & $6(6.4 \%)$ & $5(13.9 \%)$ & $1(2.7 \%)$ \\
\hline Alcohol (units/wk) & & $0.0(0.0,15.8)$ & $3.0(0.0,20.0)$ & $2.0(0.0,9.0)$ \\
\hline \multirow[t]{2}{*}{ Diagnosis } & TIA & $40(42.6 \%)$ & $11(30.6 \%)$ & $10(27.0 \%)$ \\
\hline & Stroke & $54(57.4 \%)$ & $25(69.4 \%)$ & $27(73.0 \%)$ \\
\hline \multirow[t]{4}{*}{ OCSP classification } & LACS & $27(50.0 \%)$ & $9(36.0 \%)$ & $11(40.7 \%)$ \\
\hline & PACS & $13(24.1 \%)$ & $13(52.0 \%)$ & $11(40.7 \%)$ \\
\hline & TACS & $0(0.0 \%)$ & $0(0.0 \%)$ & $0(0.0 \%)$ \\
\hline & POCS & $14(25.9 \%)$ & $3(12.0 \%)$ & $5(18.5)$ \\
\hline \multirow[t]{6}{*}{ Past medical history } & $\mathrm{AF}$ & $25(26.6 \%)$ & $6(16.7 \%)$ & $10(27.0 \%)$ \\
\hline & Diabetes & 19 (20.2\%) & $7(19.4 \%)$ & $5(13.5 \%)$ \\
\hline & IHD & $11(11.7 \%)$ & $4(11.1 \%)$ & $6(16.2 \%)$ \\
\hline & Stroke & $44(46.8 \%)$ & $12(33.3 \%)$ & $21(56.8 \%)$ \\
\hline & TIA & $36(38.3 \%)$ & $6(16.7 \%)$ & $7(18.9 \%)$ \\
\hline & Hypertension & $53(56.4 \%)$ & $20(55.6 \%)$ & $25(67.6 \%)$ \\
\hline Rockwood Frailty Score & & $4.0(3.0,6.0)$ & $5.0(4.0,6.0)$ & $6.0(4.5,6.0)$ \\
\hline MoCA & & $27.4(1.4)$ & $23.4(1.4)$ & $23.2(1.5)$ \\
\hline \multirow[t]{2}{*}{ Clinic BP (mmHg) } & Systolic & $147.3(20.5)$ & $148.1(21.0)$ & $145.2(19.5)$ \\
\hline & Diastolic & $79.6(10.5)$ & $78.9(11.5)$ & $81.8(12.5)$ \\
\hline Total Cholesterol (mmol/L) & & $4.9(1.2)$ & $4.9(1.2)$ & $4.6(1.4)$ \\
\hline Heart rate (beats per $\min )^{a}$ & & $76.6(18.9)$ & $75.9(16.8)$ & $80.4(10.2)$ \\
\hline On anticoagulation ${ }^{a}$ & & 10/25 (40.0\%) & $3 / 6(50.0 \%)$ & $3 / 10(30.0 \%)$ \\
\hline $\mathrm{HbA} 1 \mathrm{C}(\mathrm{mmol} / \mathrm{mol})^{\mathrm{b}}$ & & $52.5(47.3,69.5)$ & $49.5(43.0,82.3)$ & $73.0(51.8,106$ \\
\hline
\end{tabular}

Data presented are mean (SD), median (IQR), or frequency (\%)

${ }^{a}$ Only those with AF

${ }^{\mathrm{b}}$ Only those with diabetes

\section{Results}

Trial recruitment ran from November 2015 to July 2017, with final follow-up completed 12 months later. This was the pre-planned trial duration. Seven hundred and sixty-seven patients were screened, with 167 (21.8\%) providing consent to participate (Fig. 1). Ninety-four participants were included in the observational cohort and 73 were allocated to the RCT, 37 being randomised to intervention and 36 to control. Of the remainder screened $362(47.2 \%)$ patients were ineligible and 238 (31.0\%) were eligible but declined to participate. Of those declining to participate 18/238 (7.6\%) had a MoCA score $\geq 26,50 / 238(21.0 \%)$ had a MoCA score between 20 and 25, and 170/238 (71.4\%) had not completed cognitive testing at the time of screening. Demographic details are presented in Table 1. Both randomised groups were well matched in most areas.

During follow-up 35/73 (47.9\%) randomised participants withdrew (25 [71.4\%]) or were lost to follow-up (10 [28.6\%]), 14/36 (38.9\%) from the control group and $21 / 37(56.8 \%)$ from the intervention group. The majority of participants $(29 / 35[82.9 \%])$ not completing the trial withdrew consent to continue. Of the remainder, three died and three were withdrawn by the trial team due to a deterioration in health. Participants were not required to provide a reason for dropping out, but recurring reasons offered included 'deteriorating health' and 'not wishing to travel to the hospital for follow-up visits'.

Average MoCA scores declined significantly in the observation cohort $(-1.7$ points $[95 \% \mathrm{CI}-2.3$ to -1.1 , 
Table 2 Rates of control for secondary prevention measures by study group

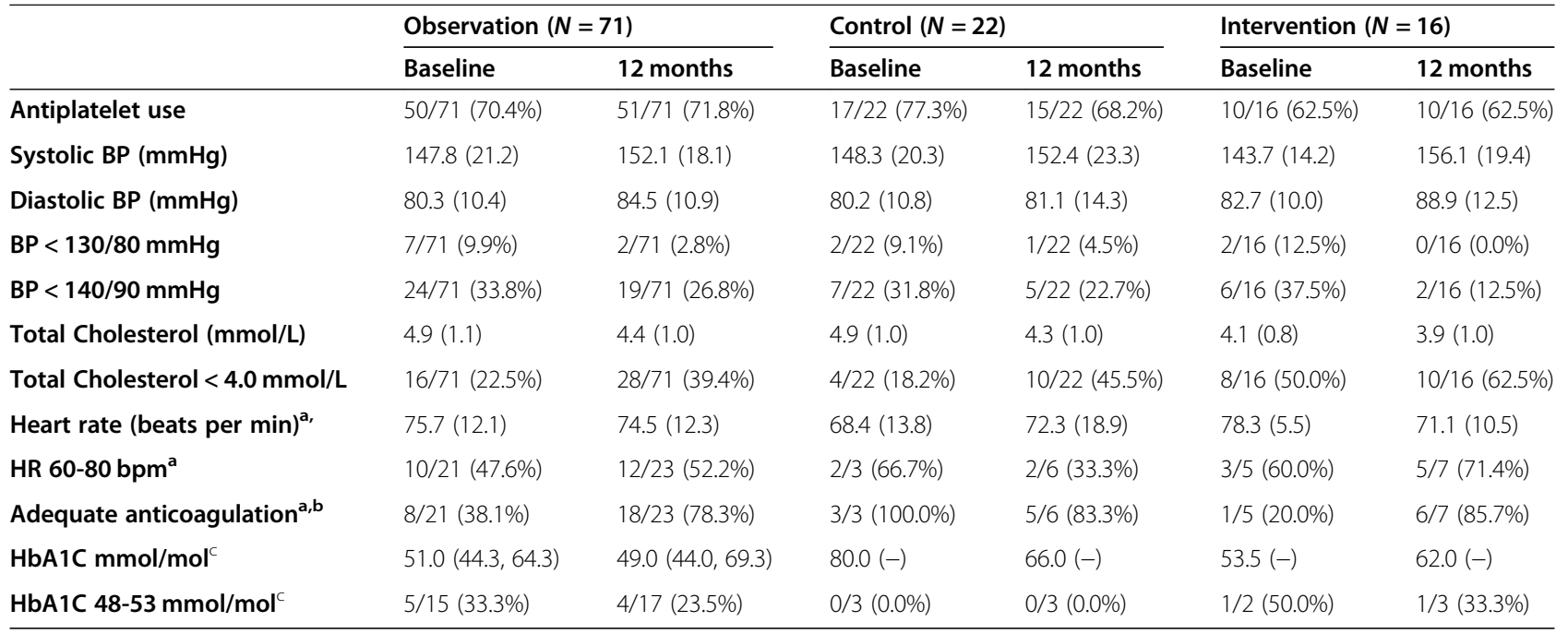

Average values and rates of control for secondary vascular prevention measures at baseline and 12 months by study group (restricted to participants who completed follow-up). Data presented are mean (SD), median (IQR), or frequency (\%)

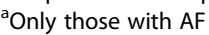

IINR 2.5-3.0 or on a DOAC

'Only those with diabetes

$p<0.0001]$ ), but not in the intervention $(-0.6$ points [95\%CI -2.3 to $1.1, p=0.45])$ or control groups $(-0.5$ points $[95 \% \mathrm{CI}-2.1$ to $1.1, \mathrm{p}=0.45]$ ). From the general linear model to estimate the effect of the intervention the mean 12 month MoCA for the Intervention group was 0.664 units lower than for Control, with $95 \%$ confidence interval for the difference (intervention minus control) being -2.69 to 1.37. Baseline rates of control for all risk factors were low across all trial groups, irrespective of BP threshold value (Table 2 and Additional file 1). There were improvements in the rates of control for cholesterol and adequate anticoagulation in all trial groups at 12 months, however, BP control rates had declined and no changes were seen in relation to heart rate and HbA1C (Additional file 2). The proportions of participants on treatment for the selected risk factors were largely unaltered after 12 months, with the exception of increases in statin use and the prescription of anticoagulants. Rates of adverse events and recurrent stroke were similar between the randomised groups (Additional file 3). Median baseline frailty scores were lower in those who completed the trial compared to those who did not (median 4.0 [IQR 3.0, 6.0] and 5.0 [IQR 4.0, 6.0] respectively, $p=0.05$ ).

\section{Discussion}

At present it is unclear whether control of multiple vascular risk factors can prevent further cognitive decline in vulnerable patients with a recent cerebrovascular event $[5,9,18]$. SERVED Memory aimed to test the feasibility of conducting such multimodal, guideline based, risk factor management in a pragmatic trial combining primary and secondary care input. We demonstrated a recruitment rate of $>20 \%$ of patients screened, suggesting that recruitment of patients with $\mathrm{MCI}$ associated with cerebrovascular disease to such a trial is possible. Although short of the recruitment target, the numbers entering the trial support its feasibility, especially given the proportion of patients with a MoCA score 20-25, or unknown at the point of screening, who declined to participate. However, nearly half of participants in the RCT arms did not complete follow-up, with this retention difficulty being partly related to frailty status. Alterations to the protocol may alleviate these difficulties, for example carrying out trial visits in the patients' homes, using online assessments, or treatment changes being made directly by the trial team rather than relaying information to the GP. Such supported self-management strategies are deliverable in this patient population as evidenced by the TEST-BP trial [19], but these changes would inevitably increase the complexity and cost of conducting the trial. Additionally, although this trial was supported by a GP applicant, more involvement of primary care in future trial design would be valuable to explore why interventions were not being implemented.

In terms of the secondary objective of assessing the effect of the intervention we did not show a betweengroup difference in change in MoCA score over 12 months. Interestingly a greater decline in cognitive scores was seen in the observational cohort. These findings are in keeping with the results of two similar trials in patients with recent stroke but no early cognitive decline. Firstly, Ihle-Hansen et al. $(N=195)$ demonstrated 
no difference in incident cognitive impairment or dementia at 12 months with a multimodal intervention compared to usual GP care [20]. Secondly, Matz et al. $(N=202)$ reported no significant difference in cognitive test scores at 24 months between those treated with a multimodal vascular risk factor intervention and usual care [21]. Conversely, two larger trials in general populations have demonstrated that similar interventions can reduce cognitive decline and the risk of requiring longterm institutional care [22, 23]. Given the small sample size and short follow-up duration of all three existing studies in stroke patients, further trials may be warranted.

The main strength of this trial is the enrolment of patients with early cognitive decline, who are at increased risk of developing dementia this preventive strategy has not previously been assessed. A further strength is the use of a pragmatic real-world design, although this also served to highlight challenges in the optimisation of care for secondary stroke prevention that would need addressing in any future trial. A limitation is that we did not consult GP's directly as to why targets were not being met, but it may reflect ongoing debate about the most appropriate risk factor targets (especially in older patients) $[6,7,24]$, or excessive demands from the existing primary care workload. Assessment of the secondary trial objectives was also limited by the retention rate and small sample size. Furthermore, due to the lack of ethnic diversity in the trial population any findings may lack generalisability.

\section{Conclusions}

Although the current protocol would not be feasible to deliver a definitive multi-centre trial due to difficulties with participant retention and application of the intervention, a successful further trial may be possible with protocol alterations as discussed. In addition, the findings of the epidemiological observation cohort suggest that such a trial should include patients with normal cognition and MCI following their cerebrovascular event, as all are at risk of further cognitive decline.

\section{Supplementary information}

Supplementary information accompanies this paper at https://doi.org/10. 1186/s12877-020-01760-z.

Additional file 1: Table 1. word document; "Rates of secondary prevention control at baseline in all participants"; average values and rates of control for secondary prevention measures at baseline by study group, including participants who did not complete the trial.

Additional file 2: Table 2. word document; "Rates of vascular risk factor treatment at baseline and 12 months"; rates of vascular risk factor treatment at baseline and 12 months by study group and changes during the trial (restricted to participants who completed follow-up).
Additional file 3: Table 3. word document; "Adverse events"; rates of serious adverse events, including deaths and recurrent stroke events, by study group.

\section{Abbreviations}

TIA: Transient ischaemic attack; MCl: Mild cognitive impairment; GP: General practitioner; BP: Blood pressure; AF: Atrial fibrillation; VaD: Vascular dementia; NNUH: Norfolk and Norwich University Hospital; MoCA: Montreal cognitive assessment; RCT: Randomised controlled trial

\section{Acknowledgements}

We thank the participants of the trial for their time. We also thank our research trial nurses Maggie Langley and Laura Ford along with our lay steering committee members for their significant contributions to the completion of the trial. Additional thanks are extended to Professor Gordon Wilcock for his advice in developing the study protocol.

\section{Authors' contributions}

PKM, JFP, YKL, CF, DT and LS were involved in the original conception and design of the study. Data acquisition was conducted by GR and WJD under the oversight of PKM and JFP. WJD and LS conducted the statistical analysis for the study. WJD drafted the initial manuscript which has been revised with input from all other listed authors. The final manuscript has been reviewed by all authors and approved for submission/publication.

\section{Funding}

This work was supported by the National Institute for Health Research (NIHR) under its Research for Patient Benefit Programme [DRF-2013-06-115]. The views expressed here are those of the author(s) and not necessarily those of the NHS, the NIHR or the Department of Health.

\section{Availability of data and materials}

The datasets used and/or analysed during the current study are available from the corresponding author on reasonable request.

\section{Ethics approval and consent to participate}

This study was conducted in accordance with the Declaration of Helsinki ethical principles for medical research involving human subjects. Ethical approval for the trial was granted by the East of England Cambridge East Research Ethics Committee (ref: 15/EE/0061). All participants provided written informed consent for their involvement. Where concern was expressed during follow-up by the subject, carer or investigator as to whether they should continue in the study due to cognitive decline, and thus being able to give informed consent, the ethics committee asked for the subject to be assessed independently of the trialists by the local dementia network team and their legal guardian as to whether it was in the subjects best interest to continue in the study. In no cases during the study was this necessary.

\section{Consent for publication}

Not applicable.

\section{Competing interests}

The authors declare that they have no competing interests.

\section{Author details}

${ }^{1}$ Ageing and Stroke Medicine, Norwich Medical School, University of East Anglia, Bob Champion Research Building, James Watson Road, Norwich, UK. ${ }^{2}$ Ageing Clinical \& Experimental Research Team (ACER), Institute of Applied Health Sciences, University of Aberdeen, Aberdeen, UK. ${ }^{3}$ Norwich Medical School, University of East Anglia, Norwich, UK. ${ }^{4}$ Norfolk and Norwich University Hospital, Norwich, UK.

Received: 15 April 2020 Accepted: 8 September 2020 Published online: 17 September 2020

References

1. Livingston $\mathrm{G}$, et al. Dementia prevention, intervention, and care. Lancet. 2017;390(10113):2673-734. 
2. Pendlebury ST, Rothwell PM. Prevalence, incidence, and factors associated with pre-stroke and post-stroke dementia: a systematic review and metaanalysis. Lancet Neurol. 2009;8(11):1006-18.

3. Leys D, et al. Poststroke dementia. Lancet Neurol. 2005;4(11):752-9.

4. Guyomard V, et al. Transient ischaemic attack, vascular risk factors and cognitive impairment: a case-controlled study. Age Ageing. 2011;40(5):641-4.

5. Ligthart SA, et al. Treatment of cardiovascular risk factors to prevent cognitive decline and dementia: a systematic review. Vasc Health Risk Manag. 2010;6:775-85.

6. Wright $\mathrm{CB}$. Should hypertension be treated in late life to preserve cognitive function? Pro Side of the Argument. Hypertension. 2018;71(5):781-6.

7. Gottesman RF. Should hypertension be treated in late life to preserve cognitive function? Con Side of the Argument. Hypertension. 2018;71(5): 787-92.

8. Ettorre $\mathrm{E}$, et al. Role of cardiovascular risk factors (CRF) in the patients with mild cognitive impairment (MCI). Arch Gerontol Geriatr. 2012;54(2):330-2.

9. Dichgans $\mathrm{M}$, Zietemann $\mathrm{V}$. Prevention of vascular cognitive impairment. Stroke. 2012;43(11):3137-46.

10. Peters R, et al. Incident dementia and blood pressure lowering in the hypertension in the very elderly trial cognitive function assessment (HVVETCOG): a double-blind, placebo controlled trial. Lancet Neurol. 2008;7(8):683-9.

11. Nasreddine ZS, et al. The Montreal cognitive assessment, MoCA: a brief screening tool for mild cognitive impairment. J Am Geriatr Soc. 2005;53(4): 695-9.

12. Pendlebury ST, et al. MoCA, ACE-R, and MMSE versus the National Institute of Neurological Disorders and Stroke-Canadian stroke network vascular cognitive impairment harmonization standards neuropsychological battery after TIA and stroke. Stroke. 2012;43(2):464-9.

13. Webb AJS, et al. Validation of the Montreal cognitive assessment versus mini-mental state examination against hypertension and hypertensive Arteriopathy after transient ischemic attack or minor stroke. Stroke. 2014 45(11):3337-42.

14. Myint PK, et al. Protocol for a feasibility randomised controlled trial of screening and enhanced risk management for vascular event-related decline in memory (SERVED memory). BMJ Open. 2017;7(11):e017416.

15. National Institute for Health and Care Excellence. Hypertension in adults: diagnosis and management. 2011; Available from: www.nice.org.uk/ guidance/cg127. [cited 2017 17th July 2017].

16. Williams B, et al. 2018 ESC/ESH guidelines for the management of arterial hypertension. Eur Heart J. 2018;39(33):3021-104.

17. Kwok CS, et al. Atrial fibrillation and incidence of dementia: a systematic review and meta-analysis. Neurology. 2011;76(10):914-22

18. Duron $\mathrm{E}$, Hanon $\mathrm{O}$. Vascular risk factors, cognitive decline, and dementia. Vasc Health Risk Manag. 2008;4(2):363-81.

19. Davison WJ, et al. Does self-monitoring and self-management of blood pressure after stroke or transient ischemic attack improve control? TEST-BP, a randomized controlled trial. Am Heart J. 2018;203:105-8.

20. Ihle-Hansen $\mathrm{H}$, et al. Multifactorial vascular risk factor intervention to prevent cognitive impairment after stroke and TIA: a 12-month randomized controlled trial. Int J Stroke. 2014;9(7):932-8.

21. Matz $\mathrm{K}$, et al. Multidomain lifestyle interventions for the prevention of cognitive decline after ischemic stroke: randomized trial. Stroke. 2015;46(10): 2874-80.

22. Ngandu T, et al. A 2 year multidomain intervention of diet, exercise, cognitive training, and vascular risk monitoring versus control to prevent cognitive decline in at-risk elderly people (FINGER): a randomised controlled trial. Lancet. 2015;385(9984):2255-63.

23. Bickel $\mathrm{H}$, et al. Reduction of long-term care dependence after an 8-year primary care prevention program for stroke and dementia: the INVADE trial. J Am Heart Assoc. 2012; (4):e000786.

24. Zanchetti A, et al. Blood pressure and LDL-cholesterol targets for prevention of recurrent strokes and cognitive decline in the hypertensive patient: design of the European Society of Hypertension-Chinese Hypertension League Stroke in hypertension optimal treatment randomized trial. Hypertens. 2014;32(9):1888-97.

\section{Publisher's Note}

Springer Nature remains neutral with regard to jurisdictional claims in published maps and institutional affiliations.

Ready to submit your research? Choose BMC and benefit from:

- fast, convenient online submission

- thorough peer review by experienced researchers in your field

- rapid publication on acceptance

- support for research data, including large and complex data types

- gold Open Access which fosters wider collaboration and increased citations

- maximum visibility for your research: over $100 \mathrm{M}$ website views per year

At BMC, research is always in progress.

Learn more biomedcentral.com/submissions 